九州大学学術情報リポジトリ

Kyushu University Institutional Repository

\title{
Manganoan Leuchtenbergite from Muramatsu, Nagasaki Prefecture, Japan
}

Okamoto, Yōhachiro

Faculty of Sciences, Kyushu University

Shirozu, Haruo

Faculty of Sciences, Kyushu University

https://doi.org/10.5109/1524296

出版情報：九州大學理學部紀要：Series D, Geology. 5 (4)，pp. 185-189，1957-01-31. Faculty of Science, Kyushu University バージョン：

権利関係 : 


\section{Manganoan Leuchtenbergite from Muramatsu, Nagasaki Prefecture, Japan}

By

\section{Yōhachiro OKAMOTO and Haruo SHIROZU}

\section{Introduction}

There are several small manganese ore deposits in the crystalline schist of the Nishisonogi Peninsula, Nagasaki Prefecture. From one of these deposits at Ōishigō of Muramatsu Village were collected in 1941 light-brown chlorite, braunite, etc., and the morphological properties of the braunite were already reported (OKAмото and others, 1944). This paper is a mineralogical note on the light-brown chlorite, which is identified here as a manganoan leuchtenbergite, a mineral of the $\mathrm{Mg}$-rich chlorite group.

\section{Occurrence}

The manganese ore deposit from which the chlorite in question has been collected forms a lenticular small ore body in the sericite-quartz schist and consists mainly of braunite, piedmontite, and quartz. The chlorite occurs as veins or massive aggregates sometimes containing fine crystals of braunite. The braunite crystals, up to 3 millimeters in diameter, have c (001), p (111), $x(421), a(100)$, and $d(201)$ faces, and are frequently twined on (101). Associating with the above-mentioned essential minerals, manganese dioxide ore, alkali-feldspar, barite, etc. are found in this deposit.

\section{Physical properties}

Color is light-brown with a pinkish tinge. Under the microscope it is pale yellow without noticeable pleochroism. Cleavage (001) perfect. It forms parallel or radial aggregates of tiny laths, up to 1 millimeter in length. Optically positive with $2 \mathrm{~V}$ very small to $0^{\circ}, \mathrm{n}_{\mathrm{X}}=\mathrm{n}_{\mathrm{Y}}=1.577, \mathrm{n}_{\mathrm{Z}}=1.589, \mathrm{n}_{\mathrm{Z}}-\mathrm{n}_{\mathrm{x}}=0.012$. Specific gravity 2.68 (picnometer method). The density calculated from the cell dimensions and the chemical formula is 2.675 .

\section{Chemical composition}

The results of the chemical analysis of the chlorite free from impurities are shown in Table 1, Column 1. The chemical formula calculated from the analysis on the basis of $18(\mathrm{O}, \mathrm{OH})$ is : 


$$
\underbrace{\left(\mathrm{Mg}_{4.72} \mathrm{Mn}_{0.05} \mathrm{Fe}_{0.07}^{3+} \mathrm{Al}_{1.14}\right.}_{5.98 \text { atoms }})(\underbrace{\mathrm{Si}_{2.72} \mathrm{Al}_{\mathrm{I} .28}}_{4 \text { atoms }}) \underbrace{\mathrm{O}_{9.88} \mathrm{OH}_{8.12}}_{18 \text { atoms }}
$$

This formula agrees closely with the ideal chlorite formula and falls in the field of sheridanite neighbouring that of leuchtenbergite in the latest classification of Hey (1954). Compared with earlier analyses, however, it is leuchtenbergite rather than sheridanite, two analyses of leuchtenbergite being given for comparison in Table 1 , Columns 2 and 3 . Columns 4 and 5 in the table are the analyses of "manganese chlorite" which faintly resemble this chlorite in the mode of occurrence, manganese content, color, etc.

On referring to its chemical composition and characteristic color caused by the manganese component, the chlorite described here may well be called "manganoan leuchtenbergite."

Table 1. Chenical analyses of some Mg-chlorites

\begin{tabular}{l|c|c|c|c|c}
\hline & 1 & 2 & 3 & 4 & 5 \\
\hline $\mathrm{SiO}_{2}$ & 29.26 & 29.07 & 30.31 & 32.51 & 33.71 \\
$\mathrm{TiO}_{2}$ & n.d. & 0.32 & - & - & - \\
$\mathrm{Al}_{2} \mathrm{O}_{3}$ & 22.06 & 21.82 & 21.72 & 16.17 & 13.80 \\
$\mathrm{Fe}_{2} \mathrm{O}_{3}$ & 1.05 & 0.83 & - & 0.56 & 1.64 \\
$\mathrm{FeO}$ & none & 3.67 & 0.56 & 0.00 & - \\
$\mathrm{MnO}$ & 0.69 & - & - & 1.06 & 2.28 \\
$\mathrm{MgO}$ & 34.08 & 29.90 & 34.60 & 35.72 & 35.88 \\
$\mathrm{CaO}$ & none & 0.19 & - & 0.00 & 0.33 \\
$\mathrm{~K}_{2} \mathrm{O}$ & n.d. & tr. & - & 0.51 & - \\
$\mathrm{Na} 2 \mathrm{O}$ & n.d. & tr. & - & 0.96 & - \\
$\mathrm{H}_{2} \mathrm{O}+$ & 13.11 & 10.76 & 13.30 & 12.59 & 13.11 \\
$\mathrm{H}_{2} \mathrm{O}-$ & 0.08 & 2.76 & - & 0.12 & \} \\
\hline
\end{tabular}

1. Manganoan leuchtenbergite from Muramatsu, Nagasaki Prefecture, Japan. Color lightbrown with a pinkish tinge. Analyst: H. SHIrozu (this paper).

2. Leuchtenbergite from the Wanibuchi mine, Shimane Prefecture, Japan. Analyst: T. Sakamoto (Sudo, 1954).

3. Leuchtenbergite from Midongy, Madagascar. Analyst: J. Orcel (Orcel, 1927).

4. Manganese-chlorite from Långban, Sweden. Color orange-red. Analyst: H. von EckerMaNN (von EckermanN, 1927).

5. Manganchlorit from Harstigen, Vermland, Sweden. Color red. Analyst: A. HamberG (HAMBERG, 1890).

\section{X-ray powder pattern}

The $\mathrm{x}$-ray powder data for $\mathrm{FeK}_{a}$ are compared with those of some Mg-chlorites with a close agreement between them as shown in Table 2. The indexing of reflections and the calculation of spacings have been done on referring to the results 
Table 2. X-ray powder data for Mg-chlorites

\begin{tabular}{|c|c|c|c|c|c|c|c|c|}
\hline \multirow{2}{*}{ No. } & \multirow{2}{*}{ Probable indices } & \multicolumn{3}{|c|}{$I$} & \multicolumn{2}{|c|}{2} & \multicolumn{2}{|c|}{3} \\
\hline & & $\mathrm{d}(\AA \AA \AA)_{\text {calc. }}$ & $\mathrm{d}(\AA)_{\text {obs. }}$ & $I$ & $d(k X)$ & I & $d(\mathbf{k x})$ & I \\
\hline 1 & 001 & 14.18 & 14.1 & 7 & 13.138 & 6 & 13.678 & 7 \\
\hline 2 & 002 & 7.09 & 7.07 & 9 & 6.970 & 9 & 7.040 & 8 \\
\hline 3 & 003 & 4.727 & 4.72 & 8 & 4.678 & 9 & 4.680 & 9 \\
\hline 4 & 020 & 4.605 & 4.59 & 1 & & & & \\
\hline 5 & 004 & 3.545 & 3.54 & 10 & 3.515 & 10 & 3.509 & 10 \\
\hline 6 & 005 & 2.836 & 2.84 & 5 & 2.824 & 5 & 2.828 & 7 \\
\hline 7 & 131,202 & 2.580 & 2.58 & 3 & broad & 8 & 2.578 & 7 \\
\hline 8 & $13 \overline{2}, 201$ & 2.538 & 2.53 & 6 & 2.523 & 0 & 2.542 & 5 \\
\hline 9 & $132,20 \overline{3}$ & 2.435 & 2.43 & 5 & 2.431 & 5 & 2.430 & 6 \\
\hline 10 & $13 \overline{3}, 202$ & 2.378 & 2.37 & 4 & 2.396 & 1 & 2.370 & 3 \\
\hline 11 & $133,20 \overline{4}$ & 2.254 & 2.25 & 3 & 2.251 & 3 & 2.247 & 4 \\
\hline 12 & 134,205 & 2.063 & 2.06 & 1 & & & & \\
\hline 13 & 007 & 2.026 & 2.026 & 2 & & & 2.021 & 7 \\
\hline 14 & $13 \overline{5}, 204$ & 2.001 & 2.000 & 6 & 1.998 & 8 & 1.998 & 6 \\
\hline 15 & $135,20 \overline{6}$ & 1.880 & 1.883 & 3 & 1.880 & 3 & 1.881 & 4 \\
\hline 16 & $13 \overline{6}, 205$ & 1.822 & 1.825 & 3 & 1.818 & 3 & 1.825 & 4 \\
\hline 17 & $136,20 \overline{7}$ & 1.712 & 1.73 & $\frac{1}{2} B$ & 1.688 & 2 & 1.706 & 3 \\
\hline 18 & $13 \overline{7}, 206$ & 1.661 & 1.660 & 1 & 1.655 & 2 & 1.659 & 2 \\
\hline 19 & $137,20 \overline{8}$ & 1.564 & 1.562 & 4 & 1.563 & 7 & 1.562 & 10 \\
\hline 20 & $060,33 \overline{1}$ & 1.535 & 1.534 & 7 & 1.531 & 9 & 1.534 & 10 \\
\hline 21 & 062,331,33̄ & 1.500 & 1.500 & 2 & 1.499 & 3 & 1.502 & 2 \\
\hline 22 & $063,332,33 \overline{4}$ & 1.460 & 1.458 & 1 & 1.455 & 2 & 1.460 & 2 \\
\hline 23 & 0010 & 1.418 & 1.417 & 2 & 1.420 & 1 & 1.417 & 3 \\
\hline 24 & $064,333,33 \overline{5}$ & 1.409 & 1.407 & $\frac{1}{2}$ & & & & \\
\hline 25 & $13 \overline{9}, 208$ & 1.393 & 1.392 & 5 & 1.394 & 8 & 1.390 & 10 \\
\hline 26 & $065,334,33 \overline{6}$ & 1.350 & 1.349 & $\frac{1}{2}$ & & & 1.350 & 2 \\
\hline 27 & $400,139,20 \overline{1} \overline{0}, 26 \overline{2}$ & 1.319 & 1.317 & 2 & 1.315 & 3 & 1.319 & 4 \\
\hline 28 & $401,26 \overline{3}$ & 1.299 & 1.297 & $\frac{1}{2}$ & & & & \\
\hline 29 & $066,335,337$ & 1.287 & 1.287 & 2 & 1.287 & 3 & 1.283 & 4 \\
\hline 30 & & & & & & & 1.251 & $\frac{1}{2}$ \\
\hline 31 & $1310,201 \overline{1}$ & 1.219 & 1.220 & 2 & & & 1.218 & 7 \\
\hline 32 & $13 \overline{1} \overline{1}, 2010,26 \overline{6}, 404$ & 1.189 & 1.190 & $\frac{1}{2}$ & & & 1.191 & $\frac{1}{2}$ \\
\hline 33 & 0012 & 1.182 & 1.182 & 1 & & & 1.181 & 3 \\
\hline 34 & $1311,20 \overline{1} \overline{2}$ & 1.132 & 1.132 & 1 & & & 1.131 & 4 \\
\hline 35 & $26 \overline{8}, 406$ & 1.096 & 1.096 & 1 & & & 1.094 & 4 \\
\hline 36 & $0610,339,33 \overline{1} \overline{1}$ & 1.042 & 1.042 & 2 & & & 1.040 & 5 \\
\hline 37 & $13 \overline{1} \overline{3}, 2012,268,40 \overline{1} 0$ & 1.032 & 1.032 & 2 & & & 1.030 & 5 \\
\hline 38 & 0014 & 1.013 & 1.013 & 2 & & & 1.013 & 3 \\
\hline 39 & & & 1.000 & 1 & & & 1.001 & 3 \\
\hline 40 & & & .988 & 2 & & & .987 & 4 \\
\hline
\end{tabular}

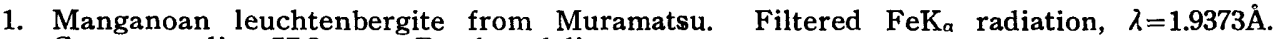
Camera radius $57.3 \mathrm{~mm}$. B: broad line.

2. Leuchtenbergite from Philipsburg, Montana. Unfiltered FeK radiation. Camera radius $57.3 \mathrm{~mm}$. Lines for $\mathrm{K}_{\beta}$ omitted. (McMurchy, 1934).

3. Sheridanite from Miles City, Montana. Unfiltered $\mathrm{CuK}$ radiation. Camera radius 57.3 $\mathrm{mm}$. Lines for $\mathrm{K}_{\beta}$ omitted. (McMorchy, 1934). 
obtained by BRINDLEY and others (BRINDLEY and ROBINSON, 1951) that the successive 1 ayer displacements were $-a_{0} / 3$ and $b_{0} / a_{0}$ was $\sqrt{ } \overline{3}$. The cell dimensions obtained here are:

$$
\mathrm{a}_{0}=\mathrm{b}_{0} / \sqrt{3 .}, \mathrm{b}_{0}=9.21 \AA, \mathrm{c}_{0} \sin \beta=\mathrm{d}_{(001)}=14.18 \AA, \beta=97.1^{\circ} \text {. }
$$

The details of the $x$-ray investigation together with those of other chlorites found in Japan will be presented in future.

\section{Differential thermal analysis}

The differential thermal analysis curve is given in Fig. 1, which shows the characteristic features of minerals of the chlorite group. Endothermic peaks are shown at about $675^{\circ} \mathrm{C}$ and $840^{\circ} \mathrm{C}$, while exothermic peaks at about $870^{\circ} \mathrm{C}$ and $920^{\circ} \mathrm{C}$. The material heated at about $1000^{\circ} \mathrm{C}$ gives an $\mathrm{x}$-ray pattern of a mixture of olivine and spinel.

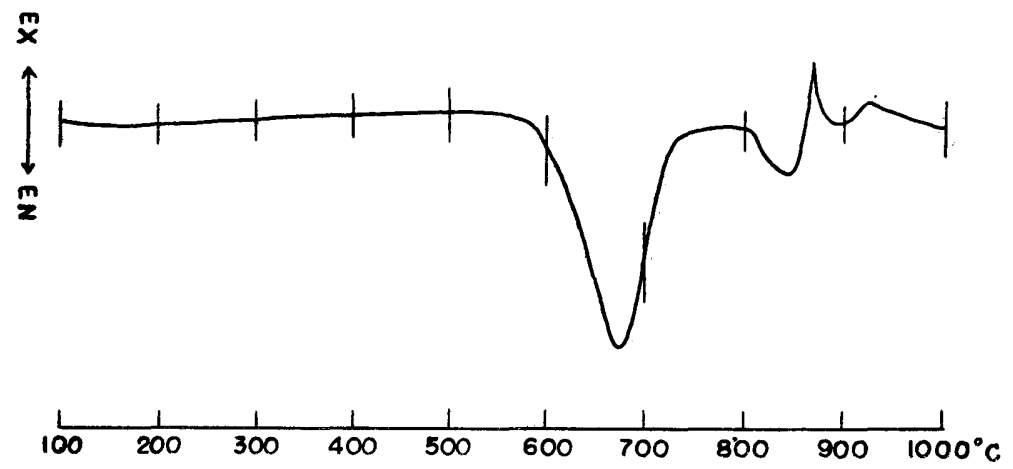

Fig. 1. Differential thermal analysis curve for manganoan leuchtenbergite from Muramatsu.

\section{Acknowledgments}

We tender our warmest acknowledgment to Professors T. Tomita and T. YoshIMURA of Kyushu University for their valuable suggestions and critical readings of this paper in manuscript. 


\section{References}

Brindeex, G. W. and Robinson, K. (1951). The chlorite minerals. X-ray identification and crystal structures of clay minerals, chap. VI, 173.

Eckermann, H. von (1927). A new manganese-chlorite from Långban. Geol. För. Stockholm Forh., 49, 450.

EckermanN, H. von (1944). The manganese-chlorite from Långban, some confirmative data. Geol. För. Stockholm Förh., 66, 721.

HAMberg, A. (1890). Ein manganhaltiger Chlorit von Harstigen. Geol. För. Stockholm Förh., 12, 580 .

Hey, M. H. (1954). A new review of the chlorites. Min. Mag., 30, 277.

Hutron, C. O. (1936). Mineralogical notes from the University of Otago. Trans. Roy Soc. New Zealand, 66, 35. [Ref. Min. Abs. 6, 364.]

McMurohy, R. C. (1934). The crystal structure of the chlorite minerals. Zs. Kr., 88, 420.

Окамото, Y., SakuraI, K., and KawaI, S. (1944). Braunite from Muramatsu Village, Nagasaki Prefecture (in Japanese). Kenkyu Hōkoku, 1, 6, published by Nippon Kobutsu Shumino-kai, Kyoto.

OrCEL, J. (1927). Recherches sur la composition chimique des chlorites. Bull. Soc. Fr. Min., 50, 75.

Supo, T. (1954). Types of clay minerals closely associated with metalliferous ores of the epithermal type. Sci. Rep. Tokyo Kyoiku Daigaku (Tokyo University of Education), C, 3, 173. 